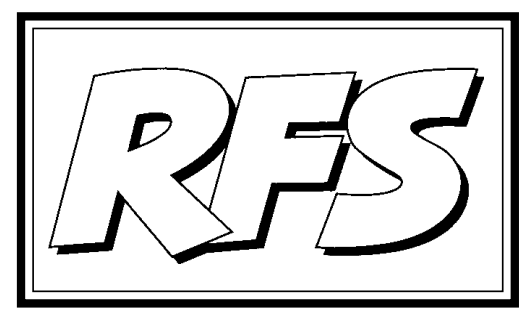

Revista de Fomento Social, 58 (2003), 151-179

\title{
La doctrina moral sobre la guerra. Su aplicación al caso de la intervención militar en Irak
}

Ildefonso CAMACHO LARAÑA, S. J .*

Cuando comienzan a redactarse estas páginas parece que la intervención armada anglo-norteamericana en Irak ha concluido. Ha sido una guerra relámpago. Pero ello no anula los costes que ha acarreado, humanos y de otros tipos, ni despeja las incógnitas sobre lo que va a ocurrir a partir de ahora en ese país. En todo caso, lo que aquí nos proponemos no es un análisis político de la guerra o dela postguerra ${ }^{1}$. Nuestro objetivo es otro. Porque dicha intervención armada ha sido una ocasión para volver a poner sobre el tapete todo lo relativo a la doctrina moral sobre la guerra: ¿puede justificarse esta acción bélica en Irak? ¿qué criterios tenemos para llegar a un juicio en este punto?

* Profesor de Pensamiento Social Cristiano en la Facultad de Teología de Granada y en la Facultad de Ciencias Económicas y Empresariales, ETEA, de la Universidad de Córdoba.

1 Sobre los problemas previsibles en la postguerra véase el documento de INTERMÓN OXFAM, "La reconstrucción de Irak y el papel de Naciones Unidas", que publicamos en este mismo número de nuestra Revista. 
Estas preguntas no son superfluas, porque la de Irak no ha sido la única guerra de nuestros tiempos, ni desgraciadamente se prevé que vaya a ser la última. Volver, por consiguiente, sobre la moral de la guerra no resultará superfluo. Ello nos dará la oportunidad de constatar la importante evolución que ha sufrido el tema en el curso de los últimos siglos, donde hasido decisiva la tradición moral, y más recientemente también el desarrollo del Derecho internacional (sobre todo, desde la conclusión de la segunda guerra mundial). Por eso comenzaremos exponiendo esta doble dimensión del tema de la guerra (el más estrictamente moral y el del Derecho Internacional) para hacer luego algunas aplicaciones al caso de la guerra de Irak.

\section{La doctrina moral de la guerra justa y su evolución}

La simple expresión "Doctrina sobre la guerra justa" causa prevención, cuando no abierto rechazo en muchos, que se preguntan perplejos: ¿cómo es posible aplicar el calificativo de "justa" a una guerra? Pero no hay que dejarse llevar por esta comprensible reacción. La complejidad que encierra la guerra y sus diferentes formas a lo largo de la historia nos obligan a estudiar con más detenimiento el tema. Por lo pronto, y para salir al paso de las reservas citadas, ténganse en cuenta estas dos consideraciones: en primer lugar, que la realidad de la guerra no ha sido siempre la misma, dependiendo de las armas disponibles y de las estrategias bélicas al uso; en segundo lugar, que lo que esa doctrina de la guerra justa ha pretendido precisamente era evitar que cualquier guerra fuese considerada legítima (por tanto, más que facilitarla, lo que ha hecho ha sido restringirla). Es más, podemos adelantar que la citada doctrina ha ido volviéndose cada vez más restrictiva y haciendo más difícil la justificación de la guerra.

\subsection{Guerra justa y Estado moderno}

La doctrina sobre la guerra tiene un larga historia. Pero recibe un nuevo impulso en la época moderna con la aparición de los Estados. Cuando existía una autoridad superior a los príncipes, siempre cabía la posibilidad de recurrir a ella en caso de conflicto. Dicha autoridad no intervenía de modo frecuente en la vida interna de las sociedades, pero quedaba como instancia última para estos momentos de crisis. Es cierto que la eficacia de esta autoridad (el Emperador o, en su caso, el Papa) fue muy variable a lo largo de los siglos, dependiendo en gran parte del poder efectivo que tenía. Pero, en 
estricto derecho, la instancia existía. Esto es lo que cambia con la aparición del Estado moderno.

Porque el Estado moderno reivindica, ante todo, su soberanía: en virtud de ella, no sólo no comparte el poder supremo con ninguna otra institución dentro de su territorio (señores u obispos, a los que se había ido privando de las potestades de que gozaban en el mundo antiguo), sino que no acepta someterse a ningún otro poder exterior. Los eventuales conflictos entre Estados tienen ahora difícil solución, porque se ha cerrado ya la posibilidad de recurrir a un poder dirimente que esté por encima de las partes enfrentadas: es el precio de la soberanía estatal. Siempre cabe negociar entre iguales (iguales, en cuanto que dotados de la misma soberanía); pero, cuando la negociación fracasa, se plantea ineludiblemente el recurso a la violencia. Estamos, pues, ante un caso de guerra. ¿Quécriterios pueden invocarse para justificar una guerra entre Estados soberanos?

Atrás quedan, por tanto, las guerras contra los infieles, "contra el turco", para recuperar los llamados "santos lugares", que eran iniciativas de la cristiandad patrocinadas por la misma sede pontificia. Y atrás quedan las guerras de religión, que están en el origen de la descomposición de la cristiandad y llegan a tales grados de crueldad que se impone la convicción de que la religión ha perdido la capacidad para mantener la paz entre los pueblos. Todo ello favorece el advenimiento y la consolidación del Estado moderno laico, que se erige así en el garante de la paz.

Pero la doctrina moral sobre la guerra había comenzado a elaborarse muchos siglos antes. Dentro de la tradición cristiana, Agustín (siglo IV) y Tomás de Aquino (siglo XIII) van a ser los hitos fundamentales hasta la llegada de la Edad moderna². Y ya en los albores de esta serán los teólogos moralistas del Siglo de Oro, especialmente Francisco de Vitoria y Francisco Suárez, los que den al tema un enfoque más acorde con la realidad del Estado.

Sin embargo, no vamos a poder entrar en las aportaciones de cada uno de ellos, ni recorrer los avances que se dieron en siglos posteriores. Vamos a optar por una visión más de conjunto presentando de forma esquemática las condiciones que se exigen para una guerra justa

Buscando una síntesis de todos ellos que nos permita una formulación lo

2 Cf. L. S. CAHILL, (2001), "La tradición cristiana de la guerra justa: tensiones y evolución", Concilium n. 290 (abril) 81-92. 
más completa posible, cabe distinguir en ella, como hiciera ya Francisco de Vitoria, dos cuestiones: los requisitos para que se pueda justificar una guerra (ius ad bellum: derecho a declarar una guerra) y las condiciones para que su desarrollo sea aceptable (ius in bello: forma de proceder en ella y medios a utilizar). El primer aspecto se refiere al hecho en sí (al fin); el segundo, a los medios. Analicemos uno y otro por separado ${ }^{3}$.

Para el ius in bellum se exigen al menos tres condiciones, que luego se completan con otras. Las tres condiciones esenciales son:

$1^{\text {a }}$ Autoridad legítima: la guerra no puede ser declarada por cualquiera, sino por la autoridad del Estado. Cualquier otro conflicto que surge en el seno de un Estado debe ser resuelto mediante la intervención de la autoridad competente dentro de ese Estado, queestá dotado de medios para ello, puesto que a él corresponde velar por la paz en el interior y entre los ciudadanos que lo integran. Ahora bien, cuando el conflicto es entre Estados, no existe una instancia a quien acudir, en el supuesto, ya indicado, de un mundo de Estados soberanos e iguales.

2a Causa justa: en principio se considera tal la tutela de un derecho violado o afirmación deun derecho reclamado (por ejemplo, el de un país a abrir vías para el comercio). Pero la tradición ha llevado, en algunas épocas, a incluir aquí otras razones, como por ejemplo, la promoción de la fe o la lucha contra doctrinas que se consideraban erróneas (las cruzadas y las guerras de religión, ya mencionadas; 0 , más recientemente, la lucha contra el comunismo o contra el fascismo).

3a Último recurso: como siempre que hay recurso a la violencia, también aquí se exige haber agotado antes todas las vías pacíficas para la resolución del conflicto.

Con frecuencia se añaden otras condiciones que muestran el deseo de restringir lo más posible la eventualidad de la guerra. Mencionaremos tres de ellas:

4a Recta intención: se exige mantenerse en la intención que legitima la guerra y no ir más allá buscando, por ejemplo, la venganza abierta o represalias sin límites.

5a Proporcionalidad: se pide con esta condición medir los previsibles

3 Seguimos la síntesis de: E. TREvISI, (2002), "Critica alla teoria della guerra giusta", Rivista di Teologia Morale, n. 133, 23-28. 
males derivados de la guerra y compararlos con los beneficios que se seguirán de ella.

6므 Probabilidad de éxito: es casi una aplicación del principio anterior, ya que se trata sencillamente de no meterse en una guerra cuyas probabilidades de éxito son nulas.

Hasta aquí las condiciones para que la declaración de guerra pueda ser legítima. Pero la ética de la guerra busca también controlar la forma como se desarrolla. Porque, por muy legítima que sea una guerra en cuanto a su declaración, no todo está justificado luego en su desarrollo, no todo vale en ella. Tres suelen indicarse para la actuación en el curso de la guerra:

a) Ninguna vida humana debe ser sacrificada si no lo exige la legítima defensa: se presupone aquí que la vida humana es un valor moral prioritario, aunque no se excluye que pueda entrar en conflicto con otros.

b) Respeto a la persona y exclusión de la tortura, cualquiera que sea su función: está en la línea de lo que acabamos de decir sobre el valor de la vida humana, sólo que ahora no se considera que esta pueda ser subordinada a fines como la obtención de información, etc.

c) Respeto absoluto a la vida de los no combatientes: se exige distinguir entre población civil y ejército y excluir cualquier tipo de ataque contra la primera.

Como se ve, la doctrina de la guerra justa ha conseguido un alto grado de precisión en cuanto a las condiciones exigidas. Goza, además, de una fuerte coherencia. Sin embargo, eso no es suficiente para considerar tal doctrina como definitiva. La realidad cambiante de la guerra no lo permite. Y es que, si la ética quiere ser honesta con la realidad, tiene que estar dejándose interpelar por esta para revisar continuamente cómo se aplican sus grandes principios a las situaciones históricas cambiantes.

\subsection{Las nuevas condiciones de la guerra contemporánea}

En efecto, desde la segunda guerra mundial para acá las condiciones que rodean a la guerra han variado considerablemente. Se han modificado, en primer lugar, las estrategias bélicas y las armas disponibles. Pero además ha cambiado el marco político mundial, que ya no se identifica tanto con el de un conjunto de Estados soberanos e iguales. Todo esto plantea la cuestión de 
si una doctrina que se elaboró sobre una forma de entender la guerra sigue siendo válida cuando en esta se han producido cambios tan notables. Veamos algunos detalles de estos nuevos escenarios de la guerra para volver de nuevo sobre la doctrina moral de la guerra justa.

Ante todo hay que abordar las nuevas estrategias para la guerra. Es imposible entrar en los detalles de un tema, de por sí complejo y eminentemente técnico. Pero sí indicaremos lo que fue un giro importante, por sus indudables consecuencias para la moralidad de la guerra: el paso de la guerra limitada a la guerra total. Este cambio vino motivado por el desarrollo de la primera guerra mundial: fue entonces cuando se llegó a la evidencia de que los medios defensivos y las tácticas tradicionales para defenderse eran tan ineficaces que provocaban una casi total estabilización de los frentes y una paralización de la guerra. Ya en el curso de aquel primer gran conflicto mundial del siglo XX se produjeron, aunque todavía de forma esporádica, acciones dirigidas contra las poblaciones civiles, que eran fruto de la espiral de odio y violencia del momento y que mostraron cuáles podrían ser las nuevas vías para una estrategia diferente.

Concluida esta primera guerra los teóricos de la estrategia militar profundizaron un nuevo modelo que incorporaba plenamente el ataque a poblaciones civiles, como la forma más eficaz de privar de sustrato y apoyo a las fuerzas militares mismas. Era evidente, por una parte, que la guerra moderna cada vez exigía una mayor movilización de la población civil, no sólo para inscribirse en los ejércitos, sino para hacer frente a las necesidades de los frentes (potenciación de la industria de guerra, etc.). Eso podríajustificar una extensión de los objetivos bélicos, que ya no se circunscribirían a los ejércitos mismos. Por otra parte, atacar a los núcleos urbanos tenía unos efectos psicológicos que podrían ser decisivos para restar apoyo a la guerra por parte de grandes masas de ciudadanos.

Estas consideraciones llevaron a consagrar la guerra total como una alternativa mejor a la de la guerra limitada. La guerra ya no enfrentaba sólo a los ejércitos, sino directamente a los pueblos. La crueldad de la guerra se acrecienta con este giro estratégico hasta plantear interrogantes éticos nuevos y decisivos.

A estas nuevas estrategias de guerra hay que unir las nuevas armas, concretamente, las armas atómicas y nucleares, y posteriormente otras de destrucción masiva (químicas y biológicas). Como se sabe, las primeras bombas atómicas -y las únicas que se han empleado hasta el momento en 
acciones bélicas- se utilizaron para acelerar la rendición de J apón al final de la segunda guerra mundial: se lanzaron contra las ciudades japonesas de Hiroshima y Nagasaki ${ }^{4}$.

En pocos años se produjo el paso de las armas atómicas ("bomba A" o bomba de fisión) a las armas nucleares propiamente dichas ("bomba $\mathrm{H}^{\circ} \mathrm{o}$ bomba de fusión). La primera explosión de una bo mba H se produjo en 1952, pero sólo como ensayo. Dichos avances coinciden con el distanciamiento entre los Estados Unidos y la Unión Soviética, después de haber sido aliados durante la segunda guerra mundial, y explican el comienzo de la carrera armamentista, que fue una de las manifestaciones más peligrosas de la confrontación entre las dos grandes potencias en la época: la guerra fría. Sin embargo en dicha carrera pueden distinguirse dos etapas bien diferenciadas, con los años 70 como momento de la transición de una a otra.

1a Hasta comienzos de la década de 1970 la carrera armamentista estuvo orientada a aumentar la capacidad destructiva de las armas. En consonancia con ella se aplicaba la estrategia de la disuasión: la posesión de armas tan destructivas se justificaba, no para utilizarlas contra el enemigo, sino para disuadir a este de emplear las suyas. Pero esto suponía una amenaza de dimensiones incalculables para la humanidad: las dos grandes potencias estaban en condiciones de destruirse mutuamente, cualquiera que fuese la que iniciase el ataque (lo que se bautizó como estrategia de la destrucción mutua asegurada). La única garantía de seguridad residía en que, evidentemente, a ninguna de ellas le interesaba dar el primer paso: en este sentido la disuasión funcionaba con eficacia. La carrera armamentista se convierte así en un estímulo fundamental para la investigación aplicada, que exige además la absorción de enormes cantidades de recursos económicos: la industria armamentista se convierte así en pionera del desarrollo económico y en fuente de importantes intereses económicos y financieros por la cantidad de recursos que mueve.

2a - Sin embargo, llegó un momento en que ya no tenía sentido aumentar la capacidad destructiva de las armas, dado el nivel alcanzado. Esta

4 En Hiroshima la bomba fue lanzada el 6 de agosto de 1945: la ciudad se convirtió en un horno de 8 kilómetros de diámetro; en ella murieron de inmediato 100.000 personas, más otras 20.000 que morirían días más tarde como consecuencia de las quemaduras que sufrieron. Tres días después fue lanzado un nuevo artefacto atómico sobre Nagasaki (el 9 de agosto), que produjo cerca de 40.000 muertos. 
convicción supuso un cambio de orientación, que se produce a lo largo de la década de 1970. A partir de ese momento, interesa más una estrategia de respuesta flexible, que consiste en la capacidad de graduar la respuesta, con objeto de responder en cada momento en el mismo nivel del ataque sufrido. La industria armamentista se orienta entonces a la diversificación de la armas: decrece el interés por los grandes ingenios de destrucción masiva, y la cantidad empieza a ceder su puesto a la calidad y a la precisión. Las nuevas armas nucleares progresan en sofisticación y precisión: ya no se pretende provocar enormes catástrofes (la destrucción directa de núcleos urbanos, por ejemplo), sino limitar su acción a los centros neurálgicos más importantes (los lugares donde se almacenan los arsenales nucleares o los puestos de mando desde donde se dirigen todas las operaciones). Este nuevo enfoque de la estrategia nuclear se convirtió en un estímulo renovado para la investigación, con efectos similares a los ya apuntados para la etapa anterior.

Pero este camino emprendido de la carrera armamentista empezó pronto a resultar demencial. Por eso, aunque se seguía avanzando en la fabricación de nuevas armas, se comienza paradójicamente un proceso de signo contrario: el desarme. Para no romper el equilibrio de las grandes potencias basado en la posesión de armas se procede a una reducción coordinada, negociada y controlada de los arsenales. Iniciada esta vía en los años 70, llegaron a firmarse algunos compromisos para el desarme ${ }^{5}$. Pero los acontecimientos de 1989 significarían un cambio sustancial en toda esta situación, como veremos más adelante.

De todos modos, esta segunda etapa de la carrera armamentista iniciada en 1970 tiene algunos efectos muy negativos, cuyas consecuencias Ilegan hasta nosotros. El más importante es el renacimiento de la posibilidad de la guerra limitada: porque esa estrategia de respuesta flexible facilitaría una respuesta dosificada y el control de la guerra para mantenerla dentro de los límites deseados. Sin embargo, asusta pensar que dicha posibilidad es más bien teórica, dada la dificultad de detener la escalada una vez comenzada. Pero hay más: porque la menor capacidad de destrucción de estas armas

5 Las negociaciones bilaterales para el desarme entre Estados Unidos y la Unión Soviética (SALT: Strategic Arms Limitation Talks) dieron como fruto dos tratados: SALT I (aprobado en 1972) y SALT II (aprobado en 1979, pero nunca aplicado porque no fue ratificado por el Congreso de los Estados Unidos). 
hace ahora más asequible el recurso a la guerra; por otra parte, ya aspiran a poseerlas, no sólo las grandes potencias, sino otros países que se sienten especialmente vulnerables por factores internos o externos.

\subsection{La revisión de la doctrina de la guerra justa}

Estas nuevas coordenadas de la guerra moderna que hemos descrito cuestionan de lleno la aplicabilidad de la doctrina tradicional de la guerra justa, la cual se ve así sometida a una profunda revisión. Para hacernos cargo de su alcance conviene trazar los momentos más representativos de la evolución que ha seguido en este tiempo la doctrina oficial de la Iglesia, como reflejo del sentir tanto de teólogos como de expertos en Derecho internacional.

Estos cambios empiezan a explicitarse de forma inequívoca después de la segunda guerra mundial. Puesto que esta guerra ha sido consecuencia del afán desmedido de un régimen político totalitario por expandirse a costa de los pueblos vecinos, se impone buscar un modelo de convivencia internacional que excluya el recurso a la violencia, cualquiera que sea la justificación que se pretenda.

Esa va a ser la postura del papa Pio XII, que inició su pontificado poco antes de que estallara la guerra. Ya en el transcurso de la misma (sobre todo en sus radiomensajes navideños), el papa fue proponiendo distintos aspectos de una doctrina política, cuyo objetivo central era el anclaje de todo el orden político nacional e internacional en un orden moral objetivo, que estuviera más allá de la voluntad de cualquier gobernante o de cualquier pueblo, y que constituyera una base firme para el orden jurídico.

Al mismo tiempo, Pío XII restringió la doctrina sobre la guerra. En sus innumerables intervenciones muestra un rechazo categórico del primado de la fuerza sobre el derecho (oponiéndose así a las ideologías totalitarias, especialmente al comunismo y al fascismo). Pero eso no le lleva a su rechazo incondicional: admite el recurso a ella en determinadas circunstancias, siempre para garantizar el respeto al derecho (en contra ahora de las corrientes pacifistas, que se oponían siempre a su uso). Lo que sí rechaza Pío XII sin excepciones es la guerra ofensiva. Sólo admite la guerra defensiva, y con muchas restricciones, que no son sino las que ya conocemos, pero especialmente subrayadas: que la iniciativa sea del Estado, que sea el último recurso, que haya proporción entre la injusticia infligida y los daños que se puedan seguir, que haya probabilidad fundada de éxito. 
La llegada a la sede pontificia en 1958 de J uan XXIII supone un cambio de orientación significativo en la cúpula de la Iglesia. El nuevo papa, que algunos consideraron en principio "un papa de transición", mostró una voluntad decidida de actualizar a la Iglesia y de abrirla a un diálogo fecundo con el mundo contemporáneo. Entre las muestras más perdurables de esta voluntad de J uan XXIII se cuenta la encíclica Pacem in terris, documento que el papa firmó el Jueves Santo, 11 de abril de 1965, apenas dos meses antes de su muerte ( 3 de junio de ese año). Por su contenido intrínseco y por esta circunstancia temporal Pacem in terris ha pasado a ser considerado como el testamento de su autor, pero también como una de las principales aportaciones del pensamiento social oficial de la Iglesia a la sociedad del siglo XX.

En el tema que nos ocupa, la encíclica sobre la paz se hace eco de las preocupaciones que antes señalábamos para este momento histórico. Sus contenidos podemos sistematizarlos en los siguientes puntos:

10 Analiza, ante todo, la carrera armamentista y sus consecuencias para el desarrollo de los pueblos ${ }^{6}$. Reconoce que el equilibrio armamentista puede ser una garantía para la paz, pero considera esta paz demasiado precaria. Por eso exige el desarme simultáneo y controlado y rechaza las armas atómicas como un peligro para la humanidad. Propone, por fin, que el equilibrio de fuerzas como base para una paz estable sea sustituido por una confianza recíproca entre los Estados.

20 En otro lugar subraya la convicción creciente de que la diferencias no se pueden resolver ya con las armas, sino por medio de las negociaciones y convenios. Esta convicción tiene que ver con la potencia destructiva de las armas. Y formula de forma apodíctica su rechazo radical de la guerra:

Por eso, en nuestra época, que se jacta de poseer la energía atómica, resulta un absurdo sostener que la guerra es un medio apto para resarcir el derecho violado ${ }^{7}$.

3o Pero quizás sus aportaciones más ricas se refieren a la propuesta de una autoridad mundial. Su punto de partida es que la interdependencia creciente de nuestro mundo exige que este sea pensado y organizado de forma unitaria. Ello implica el concepto de bien común universal, que es el correlato del concepto tradicional del bien común, sólo que

6 JUAN XXIII, Pacem in terris, nn. 109-119.

7 Ibid., n. 127. 
aplicado a la comunidad mundial.Y, también de forma correlativa, nace aquí la exigencia de una autoridad mundial con poderes suficientes para garantizar el bien común universal. Esta autoridad tendría que ser nombrada por acuerdo entre todas las naciones, y no por imposición de unas sobre otras. Su tarea esencial ha de consistir en la protección de los derechos humanos, respetando siempre el principio de subsidiariedad. Este parte de la encíclica termina, como no podía ser menos, con una referencia laudatoria de la Organización de las Naciones Unidas, de la que se reconocen sus limitaciones, pero haciendo votos para que pueda cumplir cada vez mejor estas funciones asignadas a la autoridad mundial $^{8}$.

Sólo dos años más tarde (1965) el Concilio Vaticano II, en uno de sus documentos más emblemáticos (la Constitución Pastoral sobre la Iglesia en el mundo de hoy, Gaudium et spes) desarrollará esta misma línea de pensamiento con algunas aportaciones nuevas. La respuesta a toda esta problemática está sintetizada en la Primera Sección del capítulo 5o de la Segunda Parte lleva por título: "Evitar la guerra". La doctrina del Concilio se estructura en cuatro pasos ascendentes en cuanto a su realización?:

10 Reducir la crueldad de la guerra. El punto central aquí es la insistencia en tratados internacionales para reducir la inhumanidad de los conflictos bélicos. De todos modos, y a pesar de todos los esfuerzos por limitar el alcance de la guerra, no se puede eludir la obligación del Estado de garantizar y proteger el bienestar de los pueblos: por eso no se puede excluir, en el presente estado de cosas, la guerra como legítima defensa (nunca la guerra ofensiva).

20 Eliminar la guerra total. Este es el punto más concreto en sus formulaciones. Contiene la única condena que aparece en todo el Concilio: se condena la guerra total, entendida como ataque a poblaciones civiles. Después de recordar las posibilidades de la guerra moderna, se afirma rotundamente:

Todo esto nos obliga a examinar la guerra con mentalidad totalmente nueva.

8 Todo esto se desarrolla ampliamente en los nn. 132-141 de la encíclica. Puede verse el comentario de G. Campaninı, (2000), "Oltre la 'guerra gusta'. La stagione del Concilio Vaticano II", Rivista Teologica di Lugano, vol. 5, 423-433.

9 Se tratan sucesivamente en los nn. 79-82 del citado documento. 
Se excluye así implícitamente la doctrina de la guerra justa, a la que no hay la más mínima alusión en todo el texto. Las razones pueden comprenderse a tenor de lo comentado más arriba. Pero este silencio no deja de resultar sorprendente para quien está acostumbrado a este tipo de documentos oficiales de la Iglesia, puesto que se está ignorando una tradición doctrinal que ha tenido una amplia acogida en el pasado. Y a continuación se formula de modo solemne y preciso la condena de la guerra total:

Teniendo esto en cuenta, este Concilio, haciendo suyas las condenaciones de la guerra total expresadas por los últimos Sumos Pontífices, declara: toda acción bélica que tienda indiscriminadamente a la destrucción de ciudades enteras o de extensas regiones junto con sus habitantes, es un crimen contra Dios y la humanidad que hay que condenar con firmeza y sin vacilaciones.

Lo que se condena es claramente la destrucción masiva e indiscriminada mediante armas de efectos destructivos considerables. No se condena la posesión de esas armas, ni siquiera su uso si se garantiza algún control sobre el alcance de las mismas.

30 Reducir la carrera de armamentos. Esta no puede ser el camino de una paz auténtica. Por eso se exhorta a buscar otras vías. No quiso, sin embargo, pronunciarse Concilio sobre la mera posesión de las armas nucleares, ni sobre la estrategia de la disuasión, como hubiera sido el deseo de un número considerable de Padres.

40 Hacia una eliminación total de la guerra. Aquí se abren perspectivas mucho más extensas, en la línea de crear una verdadera comunidad internacional. Esta es, como hemos dicho, una vieja idea en la tradición de la moral cristiana, que fue promovida ya por Francisco de Vitoria y en el siglo XIX por Luigi Taparelli, y que los tratadistas de Derecho internacional sólo comenzarán a hacer suya después de la primera guerra mundial.

Este recorrido confirma cómo la doctrina sobre la guerra justa se ha hecho cada vez más restrictiva, hasta el punto que casi parece imposible concebir hoy una guerra que pueda calificarse de justa. Apenas queda en pie el caso de la legítima defensa, que se aplica, no sólo a la agresión de un Estado contra otro, sino también a la violencia masiva de los derechos de los ciudadanos, o de una parte de ellos, a manos de su propio Estado ${ }^{10}$.

10 Cf. R. Coste, (1995), “De la guerre juste à la juste défense”, Études, vol. 383, 321-332. 


\section{De la doctrina moral al Derecho internacional}

El título de esta segunda parte no debe ser interpretado como una contraposición excluyente, sino en sentido complementario. Se entenderá mejor lo que se quiere decir si se tienen en cuenta dos convicciones simultáneas, que gozan de un amplio consenso en el siglo XX, ya antes de la segunda guerra mundial.

La primera convicción se refiere al hecho de la guerra: por muy horrible y rechazable que resulte en nuestro tiempo, no es posible excluir la violencia como último recurso cuando se dan violaciones graves y masivas de los derechos humanos. No aceptar esta posibilidad es renunciar a un principio ético tan nuclear como la legítima defensa, y supone dejar el mundo en manos siempre del más fuerte. Por consiguiente esta es la paradoja: que, aunque con la doctrina de la guerra justa en la mano tengamos que declarar inmoral prácticamente cualquier guerra, no es posible elevar el principio a absoluto y afirmar "toda guerra es inmoral", porque eso equivale a dejar que se imponga siempre la ley del más fuerte ${ }^{11}$.

La segunda convicción se refiere a los Estados: cada vez se perciben más los inconvenientes de dejar en sus manos al go tan trascendental como la declaración de una guerra. La realidad de la guerra moderna junto con la terrible experiencia de las dos guerras mundiales lo han mostrado fehacientemente.

Si no podemos renunciar a la legítima defensa y nos resistimos a dejar este problema en manos de la autoridad de los Estados, ¿qué salida encontrar? Aquí es donde aparece el recurso al Derecho internacional en conexión con las propuestas éticas de una autoridad de alcance mundial y su realización desde el final de la primera guerra mundial en la Sociedad de Naciones y luego en la Organización de las Naciones Unidas.

\subsection{De la Sociedad de Naciones a la Organización de las Naciones Unidas}

La Conferencia de París, que se celebra recién concluida la primera guerra mundial, tiene como fruto principal la creación de la Sociedad de Naciones ${ }^{12}$.

11 Aquí radica la diferencia fundamental con los movimientos pacifistas: cf. C. LoETSCHER, (1996), "Une guerre juste est-elle possible?", Revue de Théologie et Philosophie, vol. 128, 339-356; I. Самасно, (1987), "Perspectivas y tareas del movimiento por la paz", Proyección, vol. 34, 39-53.

12 Es oportuno recordar aquí a KANT y su propuesta de constitución de una sociedad de naciones pacíficas. Se ocupó del tema sobre todo en su Para la paz perpetua. Proyecto filosófico, de 1795. 
Su origen concreto está en el punto 14 del Programa para la Paz propuesto por Wilson en 1918: "Se constituirá una organización general de naciones bajo tratados específicos con el fin de establecer garantías mutuas de independencia política e integridad territorial tanto a los Estados grandes como a los pequeños". Con ella se pretendía superar la antigua estructura mundial de políticas nacionales, que no había sido capaz de evitar la guerra, estableciendo un organismo que garantizase la paz internacional.

El objetivo central de la nueva organización internacional es el de impedir la guerra. Con este fin los Estados miembros se obligan a llevar a la Sociedad de Naciones todos sus conflictos y someterlos a la decisión de ésta. En caso de que estallase una guerra al margen de la Sociedad de Naciones, ésta invitaría a todos sus miembros a aplicar sanciones económicas (suspensión del comercio con la nación agresora); y si las sanciones económicas no bastasen para poner término a la guerra, la Sociedad de Naciones podría ordenar una operación militar.

Como se ve, con la Sociedad de Naciones se transfiere a un organismo, que está por encima de los Estados soberanos, el poder de declarar una guerra. Estamos en la línea antes apuntada de una autoridad mundial con poderes efectivos, aunque limitados a esta cuestión y compatibles con el respeto a la soberanía de los Estados: por eso, sólo se aplica a aquellos países que han aceptado el Tratado fundacional a través de un acto explícito de soberanía. Y ahí está también la debilidad de la institución: de hecho Estados Unidos nunca fue miembro de la Sociedad de Naciones, y Alemania, que se había incorporado en 1926, decidió abandonarla en 1933.

La segunda gran conflagración mundial supone el fracaso definitivo de la Sociedad de Naciones, pero no la renuncia a un nuevo organismo que consiguiera garantizar lo que aquel primero no consiguió. Y esto es lo que se va a buscar cuando todavía la guerra no había concluido en la Conferencia de San Francisco (abril 1945), donde nace la Organización de Naciones Unidas. En la Carta de San Francisco se formulan como objetivos de la ONU: ya no sólo la resolución de los conflictos (como hiciera la Sociedad de Naciones), sino la construcción de la paz, mediante la cooperación entre los pueblos y el respeto de los derechos humanos ${ }^{13}$.

Para Kant, la paz universal siempre fue una de sus grandes preocupaciones, una paz cuyo último fundamento no podría ser sino la ley moral.

13 Se entiende así que la primera tarea que asumiera el nuevo órgano fuera la clarificación de cuáles habían de ser esos derechos que se convirtieran en los cimientos del nuevo orden 
En relación con los conflictos bélicos, la Carta de San Francisco pone las bases para un nuevo orden. Ante todo los Estados miembros renuncian a la posibilidad de declarar o de hacer la guerra. Así lo reconocen en los principios mismos sobre los que se asienta la nueva organización:

Los Miembros de la Organización, en sus relaciones internacionales, se abstendrán de recurrir a la amenaza o al uso de la fuerza contra la integridad territorial o la independencia política de cualquier Estado, o en cualquier otra forma incompatible con los Propósitos de las Naciones Unidas ${ }^{14}$.

Y en el Capítulo VII de la Carta, que trata sobre "Acción en caso de amenazas a la paz, quebrantamientos de la paz o actos de agresión", se incluye este importante pasaje:

\begin{abstract}
Ninguna disposición de esta Carta menoscabará el derecho inmanente de legítima defensa, individual o colectiva, en caso de ataque armado contra un Miembro de las Naciones Unidas, hasta tanto que el Consejo de Seguridad haya tomado las medidas necesarias para mantener la paz y la seguridad internacionales. Las medidas tomadas por los Miembros en ejercicio del derecho de legítima defensa serán comunicadas inmediatamente al Consejo de Seguridad, y no afectarán en manera al guna la autoridad y responsabilidad del Consejo conforme a la presente Carta para ejercer en cualquier momento la acción que estime necesaria con el fin de mantener o restablecer la paz y la seguridad internacionales ${ }^{15}$.
\end{abstract}

En este artículo se deja a salvo ante todo el derecho a la legítima defensa, para los individuos o para los pueblos. Es decir, no se excluye como último recurso. Pero se añade que este derecho sólo tiene vigencia mientras el Consejo de Seguridad no ha intervenido de acuerdo con sus competencias. Luego, es al Consejo de Seguridad a quien corresponde decidir las medidas a tomar, sin excluir últimamente la violencia, en caso de conflicto entre Estados miembros.

La aplicación práctica de los principios formulados en la Carta de las Naciones Unidas y aceptados por todos los Estados miembros no ha sido fácil. La causa principal de esta dificultad no es otra que la siempre discutible neutralidad del Consejo de Seguridad. Y es que este organismo está constituido por representantes gubernamentales, una parte de los cuales posee además derecho de veto. Por estas razones el funcionamiento de dicho órgano estuvo prácticamente bloqueado durantetodo el perio do de la guerra

político internacional. La Declaración Universal de los Derechos Humanos sería aprobada el 10 de diciembre de 1948.

14 Carta de las Naciones Unidas, art. 4-2.

15 Ibid., art. 51. 
fría. Producido el colapso del comunismo pareció en un primer momento que la situación se desbloqueaba. Y es indudable que la ONU se ha mostrado mucho más activa en la década de los 90 . Pero no podría negarse que en los casos más delicados de nuevo se ha impuesto el bloqueo como consecuencia de los intereses contrapuestos de los miembros del Consejo de Seguridad, especialmente de Estados Unidos y de los otros que también disponen del derecho de veto.

Hay que reconocer que este mecanismo del Consejo de Seguridad funcionó cuando la intervención en Irak en $1991^{16}$. Entonces había precedido una resolución del Consejo de Seguridad (la n. 678, de 29 de noviembre de 1990), por la quese daba de plazo a Irak hasta el 15 deenero de 1991 para que retirara sus ejércitos del territorio kuwaití que había sido ocupado el 1 de agosto de 1990 y cumpliera todo lo que se le había exigido en una serie de resoluciones anteriores. En caso contrario, se autorizaba a los Estados miembros para intervenir con el fin de "hacer valer y llevar a la práctica las resoluciones" citadas. Como el plazo se cumplió sin que Irak hubiese respondido convenientemente, el 17 de enero de 1991 se produjo la intervención de una alianza de 32 países dirigida por Estados Unidos ${ }^{17}$.

Puede decirse, como conclusión de este apartado, que parece como si la legitimación de una guerra no se apoyara tanto en razones morales cuanto en razones jurídicas: una guerra sería justa, no porque existan argumentos morales en su favor, sino porque se ha procedido formalmente según está estipulado. ¿Resultaría, entonces, que, más que de "guerra justa", habría que hablar de "guerra legal"? Creemos que la contraposición no hay que llevarla a esos extremos. Volviendo a la idea apuntada más arriba de la complementariedad entre lo ético y lo jurídico, habría que afirmar: en todo caso, la declaración formal de guerra tendrá que apoyarse en razones éticas; pero el órgano legitimado para hacer esa declaración (y la aplicación de la doctrina moral a una situación particular) no es un Estado (que además sería juez y parte), sino un organismo del que se quiere asegurar que actúa con el mayor grado de neutralidad posible.

16 Puede verse nuestro editorial: Consejo de REDACCIÓN, (1991), "La guerra del Golfo, ¿ha terminado?", Revista de Fomento Social, vol. 46, 117-120; y también: G. Pérez Alcalá, (1991), "Los orígenes del conflicto del Golfo", ibid., 233-246.

17 La discusión sobre la justificación ética de esta intervención puede verse en: CIVILTÀ CATTOLICA, (1991), “Coscienza cristiana e guerra moderna”, Civiltà Cattolica, vol. 142/3, 3-16; J. P. LanGan, (1992), "The J ust-War Theory after the Gulf War", Theological Studies, vol. 53, 95-112. 


\subsection{Los nuevos escenarios de los conflictos: la globalización}

Para completar esta panorámica es preciso aludir todavía a los cambios derivados de los acontecimientos de 1989: en efecto, la caída del muro de Berlín ha supuesto una nueva configuración del mundo, que afecta también a la forma como se plantean y afrontan los conflictos bélicos. El fenómeno más determinante es el proceso de globalización, que se ha visto acelerado por la desaparición del bloque soviético. Las manifestaciones de la globalización son variadas e importantes. Por lo que a la cuestión de la guerra respecta, hay que señalar cuatro fenómenos que marcan muy significativamente estos albores del siglo XXI:

- En primer lugar, y como una de las consecuencias de más calado de la globalización, la reducción del poder del Estado y el cuestionamiento de su soberanía: jurídicamente dicha soberanía del Estado no es discutida por nadie, pero en la práctica cada vez resulta más débil y menos operativa. Esto explica la aceleración de los procesos de integración supraestatal -el más avanzado de los cuales es, sin duda, la Unión Europea-, que obedecen a una lógica comprensible de aunar fuerzas para situarse mejor en el escenario mundial.

- En segundo lugar, el fortalecimiento de otros poderes fácticos con mayor capacidad que muchos Estados, entre los que hay que mencionar obviamente a las grandes empresas multinacionales, que manejan con frecuencia recursos económicos superiores a los presupuestos de muchos Estados y tienen una estructura internacional que les permite actuar en contra de las políticas de muchos gobiernos.

- En tercer lugar, el comercio de armas y el tráfico ilegal de las mismas, fenómeno que tiene que ver con los intereses de empresas multinacionales y que se facilita con la liberalización de los mercados. Armar a los pueblos se convierte así en un objetivo económico de un sector de la industria, la industria armamentista. Esta es otra dimensión, económica, de la guerra que no se puede ignorar. Y sabemos que muchas veces son las naciones más pobres los clientes de dicha industria: la inestabilidad de sus gobiernos y de sus fronteras son un motivo añadido para incrementar el arsenal armamentista, reduciendo de modo paralelo recursos públicos que deberían ser asignados a otros destinos urgentes.

- Por último, hay que incluir entre estos poderes fácticos las redes del terrorismo internacional, a los que la liberalización de las fronteras 
también facilita las cosas: los atentados del 11 de septiembre de 2001 en Nueva York y Washington han dejado ver hasta dónde llega su audacia y hasta qué punto son una amenaza para la estabilidad mundial. Por eso resulta urgente actuar contra él. Pero en unas coordenadas diferentes, porque en su caso ya no se dan las condiciones básicas de la guerra moderna. No existe un Estado enemigo al que enfrentarse con un territorio propio, ni vale la estrategia de la disuasión. Lo que existe es una red difusa, que resulta casi imposible de identificar: por eso su amenaza es superior a la tradicional que pudiera provenir de un Estado, y sus efectos son más desestabilizadores y peligrosos.

\subsection{La intervención humanitaria}

Pero no es la lucha antiterrorista la única modalidad específica de la guerra hoy. Conviene recordar que en los últimos años otros muchos episodios de acción armada no son, estrictamente hablando, choques entre Estados soberanos. Las tensiones que puedan existir entre estos no siempre desembocan el conflictos abiertos. En cambio se multiplican otras modalidades de guerra, entre las que destacan las que se producen en el interior de un Estado. Unas son guerras contra el Estado, que recogen la tradición de la guerra revolucionaria: en ellas el Estado se enfrenta con una parte de los ciudadanos que lo integran. Otras son guerras entre facciones dentro de un único Estado, como consecuencia de enfrentamientos que tienen detrás una larga historia y que se reavivan en momentos en que el Estado muestra una debilidad especial (los casos de la Unión Soviética o Yugoslavia son buenos ejemplos, así como otras situaciones en África). Este tipo de situaciones es el que ha puesto nuevamente de actualidad el tema de la derecho de intervención humanitaria.

En algunos de estos casos de conflictos en el interior de un Estado la ONU ha podido ejercer sus funciones a través del Consejo de Seguridad: por ejemplo, en Somalia (1992), en Ruanda (1994) o en Bosnia-Herzegovina (1994-95), con intervenciones que contribuyeron a establecer la paz o, al menos, a reducir los efectos de las guerras. No ocurrió lo mismo con ocasión del conflicto de Kosovo (1999), cuando el Consejo de Seguridad fue incapaz de llegar a una resolución para intervenir. Ante la imposibilidad de un respaldo jurídico, la OTAN, apoyada por la Unión Europea, se decidió a intervenir, invocando para ello nuevamente motivos éticos. Estamos ante un caso de intervención humanitaria. 
La intervención humanitaria consiste en la actuación de varios países dentro de otro país, aunque este último no les amenace a ellos. La historia de la intervención humanitaria es Iarga ${ }^{18}$. Ya Grocio y Pufendorf reconocieron en el siglo XVII el derecho a recurrir a las armas para combatir la tiranía en un país vecino. Y Vitoria (ya en el siglo XVI) y Suárez la justificarían para defender a los inocentes. Más recientemente, desde finales del siglo XIX, ha sido invocada por diferentes Estados para defender a los propios nacionales en el extranjero o para actuar en otro Estado cuando los derechos son en él masivamente violados. A Antoine Rougier se debe la primera elaboración sistemática de la "intervención de humanidad", a la que se definía como: "un derecho de intervención a favor de uno o varios Estados frente a los actos llevados a cabo por un gobierno contrarios a las leyes de la humanidad"19.

Llegados a 1945, con la creación de la ONU, la intervención humanitaria parece quedar totalmente descartada. En efecto, la Carta de Naciones Unidas, no sólo incluye la renuncia por parte de los Estados a recurrir a la amenaza o al uso de la fuerza frente a o tro Estado, como hemos visto, sino que consagra además el principio de no intervención en su artículo 2-720. Si el respeto de los derechos humanos es el principal objetivo de la ONU, ¿cómo garantizarlo cuando se producen violaciones masivas y el Consejo de Seguridad, por las razones que sean, se encuentra imposibilitado para actuar?

Son importantes los autores que subrayan cómo, en una situación así, debe prevalecer la salvaguarda de los derechos humanos. En la práctica situaciones de este tipo no han dejado de darse desde 1945, por lo que la intervención de los Estados, invocando precisamente razones humanitarias, fueron numerosas ${ }^{21}$, aunque no siempre unánimemente reconocidas.

En todo caso, la intervención humanitaria sigue siendo considerada como un supuesto ético aceptable. Y la praxis ha contribuido a precisar mejor las condiciones que se exigirían para poder hablar de intervención humanitaria,

18 Para lo que sigue puede consultarse una monografía tan exhaustiva sobre el tema como es la de: J. F. Escudero Espinosa, (2002), Aproximación histórica a la noción de intervención humanitaria en el Derecho internacional, Universidad de León.

19 A. Rougier, (1910), “La théorie de l'intervention d'humanité", Revue générale de droit international public, n. 4, 468-526. Citado por Escudero Espinosa, (2002), 6.

20 El texto dice así: Ninguna disposición de esta Carta autorizará a las Naciones Unidas a intervenir en los asuntos que son esencialmente de la jurisdicción interna de los Estados, ni obligará a los Miembros a someter dichos asuntos a procedimientos de arreglo conforme a la presente Carta. 
y distinguirla de intervenciones que pueden estar motivadas por otros intereses. De este modo pueden enumerarse los siguientes requisitos para una intervención humanitaria que merezca realmente este nombre ${ }^{22}$ :

- que haya violaciones graves y masivas de los derechos humanos;

- que las víctimas soliciten la intervención extranjera;

- que la obstinación del Estado violador haya agotado todos los medios pacíficos (que estemos, por tanto, nuevamente ante un caso de último recurso);

- quela intervención la hagan varios Estados, nunca uno solo (para evitar el enfrentamiento directo entre dos Estados);

- que el motivo de la intervención sea estrictamente humanitario, y no se mezclen otros.

Volvemos, pues, a planteamientos estrictamente morales, que ponen de nuevo la decisión en manos de Estados particulares. Los riesgos mencionados más arriba para este tipo de intervención se repiten aquí. Por eso se exige que no sea un solo Estado el que intervenga, sino varios; y por eso se exigen también otra serie de condiciones bastante estrictas, que hacen pensar en la dificultad de encontrar casos en que la intervención humanitaria esté moralmente justificada. Pero todo ello pone de manifiesto otra vez que no es posible renunciar precipitadamente al derecho de legítima defensa: y que cuando los mecanismos normales (en este caso, el Consejo de Seguridad) no son capaces de actuar es preciso buscar vías alternativas de solución.

\section{Algunas aplicaciones al caso de la guerra de Irak}

Al leer muchas de las cosas que preceden, inevitablemente habrá pensado más de una vez el lector en los recientes acontecimiento de Irak. Ahora ha

21 Son casos conocidos los de los países árabes en Palestina (1948), Francia y Bélgica en el Congo (1964), Estados Unidos en Santo Domingo (1965), India el Pakistán Oriental (1971), Indonesia en Timor Oriental (1975), Sudáfrica en Angora (1975), Bélgica en el Zaire (1978), Vietnam en Camboya (1979), Tanzania en Uganda (1979), Estados Unidos en Granada (1983).

22 Cf. para lo que sigue: O. CORTEN, (2001), "La référence au droit international comme justification du recours à la force: vers une nouvelle doctrine de la 'guerre juste'?", en: A.-M. Dillens (dir.), La guerre et l'Europe, Bruxelles. Publications des Facultés Universitaires de SaintLouis, 69-94. 
llegado el momento de hacer algunas aplicaciones, que dividiremos en dos partes: en primer lugar, el planteamiento que ha hecho Estados Unidos de la intervención; en segundo lugar, los análisis que se han llevado a cabo de la intervención armada desde un punto de vista moral.

\subsection{El planteamiento norteamericano}

Indudablemente la preocupación de Estados Unidos con el régimen de Irak tiene que ver con la amenaza terrorista. Por eso ha adquirido tonos de urgencia después de los atentados de septiembre de 2001. En este sentido, el marco es diferente al de la intervención de 1991, que respondía a la invasión por parte de Irak del territorio soberano de un país vecino, Kuwait.

Por eso se ha recurrido al concepto de guerra preventiva, una categoría diferente de las tradicionales sobre las que se había elaborado toda la doctrina de la guerra: guerra ofensiva y guerra defensiva, y, más recientemente, guerra revolucionaria. Una guerra preventiva es la que se emprende para prevenir los daños que un Estado podría infligir a otro Estado o a otros Estados, privándolo de tal capacidad o posibilidad.

Este concepto de guerra preventiva se encuentra ya en algunos tratadistas recientes de la guerra, como es el caso de Michael WalzeR ${ }^{23}$. Distingue este autor dos casos muy diferentes. El primero es aquel en que habría que defenderse de una agresión que sea inminente aunque todavía no haya tenido lugar. Walzer ve justificado el ataque en este caso, pero exigiendo que se den condiciones muy estrictas: que la necesidad de defenderse sea instantánea y abrumadora, de manera que no dé lugar a la elección de medios ni haya tiempo para la deliberación. Ahora bien, frente a esta situación de peligro inminente, Walzer presenta otro, al que denomina concretamente guerra preventiva: cuando el ataque responde a un peligro lejano, tratándose entonces de una cuestión de previsión y de libre elección. En este segundo caso, de ninguna manera se justificaría éticamente la guerra.

Con estos antecedentes resulta de gran utilidad analizar un reciente documento oficial: la Estrategia de seguridad nacional de los Estados Unidos de

23 M. Walzer, (2001), Guerras justas e injustas. Un razonamiento moral con ejemplos históricos, Barcelona, Paidós. Véase especialmente el capítulo 5: "Las anticipaciones". La primera edición de esta obra en inglés se remonta a 1977. 
América ${ }^{24}$. De entrada nos interesa su capítulo 50: "Prevenir que nuestros enemigos nos ataquen a nosotros, a nuestros aliad os y a nuestros amigos con armas de destrucción masiva". En él se describe la nueva situación derivada del encuentro del radicalismo con la modernísima tecnología, que ha hecho tan peligrosos a lo que en el documento se denominan "Estados canallas y terroristas" (y entre los que se cita concretamente a dos: Irak y Corea del Norte). Esta nueva situación es muy distinta de la guerra fría y ha hecho inútil la estrategia de la disuasión basada en la posesión de armas con el fin de disuadir al enemigo potencial por temor a las represalias. Los actuales agentes del terrorismo internacional no conciben dichas armas como un último recurso, sino que aspiran a emplearlas para intimidar y para agredir militarmente a sus vecinos. De todo esto se concluye:

Dados estos objetivos, los Estados Unidos no pueden por más tiempo limitarse a confiar en una postura de reacción, como hicimos en el pasado. La imposibilidad de disuadir al atacante potencial, la inminencia de las amenazas actuales y la magnitud del daño que pueden causarnos la armas elegidas por nuestros adversarios, no permiten ya esa opción. No podemos dejar que nuestros enemigos golpeen primero ${ }^{25}$.

Y poco después se recuerda la postura del Derecho internacional ante situaciones semejantes, para añadir que esa doctrina debe ser adaptada a las condiciones actuales:

Durante siglos el Derecho internacional reconoció que las naciones no tienen que sufrir un ataque antes que ellas puedan legalmente entrar en acción para defenderse de fuerzas que representan un peligro inminente de ataque. Los especialistas en Derecho y los juristas internacionales condicionan con frecuencia la legitimidad de la anticipación a la existencia de un peligro inminente-muy frecuentemente a una movilización visible de tropas, navíos y fuerzas aéreas que se preparan para el ataque.

Tenemos que adaptar el concepto de peligro inminente a las capacidades y objetivos de los adversarios de hoy. Los Estados canallas y los terroristas no pretenden atacarnos usando medios convencionales. Saben que tales ataques fracasarían. En lugar de ello, confían en los actos de terror y, potencialmente, en el uso de armas de destrucción masiva, armas que pueden ser fácilmente encubiertas, secretamente distribuidas y usadas sin previo aviso.

Si volvemos a la distinción de Walzer, comprendemos cómo el documento

24 The National Security Strategy of the United States of America (September 2002). El documento puede consultarse en la página web de la Casa Blanca.

25 Ibid., p. 15. 
que estamos analizando se esfuerza por interpretar la actual amenaza como la situación que justificaría una guerra. La misma semejanza de los términos ingleses preemptive y preventive facilita el interpretar como preemptive war (guerra anticipatoria) lo que más bien sería preventive war (guerra preventiva), escudándose para ello en esa necesidad de revisar la doctrina tradicional de los juristas ${ }^{26}$. Entendiendo la amenaza delrak como inminente, el gobierno de Bush considera justificada la intervención armada.

Hasta ahora nos hemos referido sólo a un punto del documento sobre estrategia de Estados Unidos de septiembre 2002. Pero este texto en su conjunto ofrece un marco de indudable interés para encuadrar los planteamientos norteamericanos en relación con la intervención armada en Irak. En la introducción, firmada personalmente por el presidente Bush, se formula la pregunta sobre el papel que corresponde a Estados Unidos una vez que "las grandes luchas del siglo XX entre libertad y totalitarismo concluyeron con una victoria decisiva de las fuerzas de la libertad"27, las cuales están en perfecta sintonía con las aspiraciones profundas de todos los pueblos. Y el documento comienza con esta declaración casi programática que, a pesar de su longitud, reproducimos íntegramente:

Los Estados Unidos poseen una fuerza y una influencia en el mundo sin precedentes y sin igual. Sostenida por la fe en los principios de libertad y en los valores de una sociedad libre, esta posición conlleva responsabilidades, obligaciones y oportunidades sin par. La fuerza incomparable de esta nación debe ser usada para promover un equilibrio de poder que favorezca la paz.

Durante la mayor parte del siglo veinte, el mundo ha estado dividido por una profunda lucha ideológica: visiones totalitarias destructivas frente a libertad e igualdad.

Esta lucha ha terminado. Las concepciones militantes de clase, nación o raza, que prometían utopía y han desembocado en miseria, han quedado vencidas y desacreditadas. América está ahora menos amenazada por Estados triunfadores que por la falta de estos.

Estamos menos amenazados por flotas y ejércitos que por tecnologías catastróficas en manos de unos pocos resentidos. Y tenemos que aniquilar estas amenazas para nuestra nación, para nuestros aliados y amigos.

Este es, por tanto, un tiempo de oportunidad para América. Vamos a traducir este momento de influencia en décadas de paz, prosperidad y libertad. La estrategia de

26 Sobre esta cuestión, cf.: M. PedrazzI, (2003), “Dottrina Bush sulla 'guerra preventiva' e diritto internazionale", Aggiornamenti Sociali, n. 2, 134-138; N. SerRa I SerRA, (2003), “La militarización de la política exterior de Bush", El País, 7 de abril, 10-11.

27 The National Security Strategy..., I.C., III. 
seguridad nacional de los Estados Unidos se basará en un internacionalismo inconfundiblemente americano, que refleja la unión de nuestro valores y de nuestros intereses nacionales. El objetivo de esta estrategia consiste en ayudar a hacer al mundo no precisamente más seguro y mejor. Nuestras metas en el camino hacia el progreso son claros: libertad política y económica, relaciones pacíficas con los demás Estados y respeto a la dignidad de la persona. Y este camino no es sólo de América. Está abierto a todos.

Para alcanzar estas metas los Estados Unidos:

- serán paladines en las aspiraciones en pro de la dignidad humana;

- reforzarán alianzas para vencer al terrorismo internacional y actuarán para prevenir ataques contra nosotros y nuestros amigos;

- colaborarán con otros para desactivar los conflictos regionales;

- evitarán que nuestros enemigos nos amenacen o amenacen a nuestros aliados o amigos con armas de destrucción masiva;

- iniciarán una nueva era de crecimiento económico global mediante mercados libres y libre comercio;

- ampliarán el mundo del desarrollo abriendo las sociedades y construyendo la infraestructura de la democracia;

- desarrollarán agendas para la acción cooperativa con los otros centros principales de poder; y

- transformarán las instituciones de seguridad de América para hacer frente a los retos y oportunidades del siglo XXI28.

Estos ocho puntos enumerados constituyen los ocho capítulos en que se estructura todo el documento. Pero más que entrar en el análisis de cada uno (ya lo hemos hecho del que más nos interesaba), nuestra intención ahora es sólo subrayar los presupuestos desde el que esta estrategia de seguridad está concebida. Ante todo, el go bierno norteamericano es muy consciente de su poder inigualable en el mundo tras la caída del bloque soviético: y ese es un hecho incontestable. Más inquietante es la consecuencia que de ahí se saca: la de erigirse en promotores de un modelo de sociedad libre y abierta en todo el mundo, sin reparar en diferencias culturales o de otro tipo. Esta "misión" que los Estados Unidos se atribuyen está expresada con más crudeza en estas palabras del presidente Bush que el mismo documento recoge:

28 Ibid., 1-2. 
Cuando están en juego los valores comunes y las necesidades de los hombres y las mujeres no hay ningún desencuentro entre culturas. Los requisitos de la libertad valen plenamente también para África y para América Latina y para todo el mundo islámico. Los pueblos de las naciones islámicas quieren y merecen las mismas libertades y las mismas oportunidades que los pueblos de cualquier otra nación (...). La causa de nuestra nación ha sido siempre más grande que la defensa de nuestra nación. Luchamos, como hemos luchado siempre, por una paz justa, por una paz que promueva la libertad. Defenderemos la paz contra las amenazas de terroristas y tiranos. Preservaremos la paz construyendo buenas relaciones entre las grandes potencias. Y extenderemos la paz promoviendo sociedades libres y abiertas en todos los continentes ${ }^{29}$.

\subsection{La intervención militar en Irak desde un punto de vista ético}

Indudablemente la intervención anglo-norteamericana en Irak está muy marcada por el horror al terrorismo que con tanta fuerza atenaza al pueblo y al gobierno estadounidense tras los atentados de septiembre de 2001. Sin embargo, los intentos de legitimar la intervención son más que discutibles. Convencido de la imposibilidad de contar con el respaldo de una resolución del Consejo de Seguridad, el gobierno de Bush ha buscado argumentos en la presunta vinculación del régimen iraquí con el grupo terrorista AI Qaeda (que nunca ha podido ser probado), en la amenaza que representa dicho régimen para la estabilidad mundial en cuanto poseedor de armas de destrucción masiva (cosa que tampoco ha llegado a demostrarse), en la necesidad de liberar al pueblo iraquí y devolverlo a la normalidad democrática ${ }^{30}$.

Este último argumento, en sí mismo el más ambicioso, es el más problemático. Conecta con esa "misión" que Bush asigna a su país de promover la libertad y la democracia en todo el mundo. Nada hay que objetar al objetivo mismo. Pero es más que dudosa la viabilidad de la forma en que se pretende conseguir. Por dos razones. Ante todo, derrocar a gobierno tiránico (y nadie cuestiona que el de Irak lo sea) no conduce automáticamente a la democracia; menos aún, cuando el pueblo iraquí no ha tenido nunca en su historia una experiencia de tal régimen político. Porque la democracia es algo más que un conjunto de instituciones políticas; es además un talante interiorizado por la

29 Ibid., 1. Dichas palabras pertenecen a un discurso en West Point, Nueva York, pronunciado el 1 de junio de 2002.

30 Para la crítica que sigue puede verse el editorial: CiviLtà CATtolica, (2003), "No a una guerra 'preventiva' contro I'Iraq", Civiltà Cattolica, quaderno 3.662 (18 gennaio), 107-117. 
sociedad y que inspira toda la cultura del pueblo. Pero hay otra razón de peso para ser escépticos ante la intención norteamericana: una intervención armada no hará sino ahondar más las profundas distancias que separan al mundo islámico de Occidente en general, y más particularmente de Estados Unidos. Sin entrar en los detalles de otros conflictos abiertos (entre los que el problema palestino-israelí no es el menor), hay razones para temer que esta nueva intervención acabe ahondando las heridas y contribuyendo a una mayor inestabilidad mundial. Sólo en el marco de estas hondas confrontaciones históricas y con la voluntad decidida de abordarlas en sus últimas raíces, y no sólo en sus manifestaciones por muy graves que sean, puede afrontarse con realismo el problema del terrorismo internacional.

Pero es el concepto mismo de guerra preventiva y su aplicación lo que plantea problemas más serios. Ya hemos aludido a la confusión entre guerra preventiva y guerra anticipatoria y al intento de legitimar aquella con los argumentos que, en todo caso, sólo valdrían para esta segunda. La crítica a esta modalidad de guerra puede hacerse desde dos puntos de vista complementarios: desde el concepto mismo y desde la aplicación de la doctrina tradicional de la guerra justa.

El concepto de guerra preventiva es altamente problemático por las cuestiones que plantea. ¿Quién determina si se da esa situación? ¿el Estado que siente la amenaza? ¿será ese el mejor juez, siendo a la vez parte, y en algo donde juegan tan fuertemente sentimientos incontrolables y fácilmente manipulables? Si dejamos en manos del Estado amenazado esta decisión ¿no estamos volviendo a la situación en que cada Estado es la instancia adecuada para declarar la guerra? Resultaría oportuno recordar aquí aquel sabio principio de Kant - "actúa en cada momento de modo que la máxima que aplicas en tu vida pueda convertirse en principio universal"-: ¿hasta dónde llegaría la inestabilidad en el mundo si cada Estado estuviera legitimado para desencadenar un ataque armado contra otro Estado sólo cuando juzgase que está amenazad o por él?Y, por fin, el peligro de justificar muchos ataques como guerra "preventiva" ¿no nos llevaría sin más a guerras "ofensivas", recuperando un concepto cuya justificación ética quedó hace tiempo excluida?

Han sido muchas las manifestaciones que se han producido en el seno de la Iglesia católica y de las Iglesias cristianas en general, en estos últimos meses: y es notable, y hasta inusitado, el consenso en torno a un rechazo de esta intervención armada. De entre todos ellos destacan las manifestaciones 
de los obispos norteamericanos ${ }^{31}$. Es en ella donde se recurre nuevamente a la doctrina clásica de la guerra justa para aplicarla a la guerra preventiva. Subrayan cuatro condiciones:

A) Causa justa. Según la expresión del Catecismo de la Iglesia católica, la causa es justa cuando "el daño causado por el agresor a la nación o a la comunidad de naciones sea duradero, grave y cierto". Esta formulación es considerada, en sí misma, como incompatible con el concepto mismo de guerra preventiva.

B) Autoridad legítima. Habría que atenerse a los imperativos constitucionales de Estados Unidos, a un amplio consenso dentro de la nación, a algún tipo de respaldo internacional (dentro del marco de la ONU). Y habría que considerar las consecuencias para la población iraquí y para la estabilidad regional y mundial.

C) Probabilidad de éxito y proporcionalidad. Son imprevisibles las consecuencias de esta intervención, no sólo para Irak, sino también para la paz y la estabilidad en todo el Medio Oriente. Porque el uso de la fuerza puede provocar ataques del tipo que se quieren evitar y pueden provocar daños considerables a la población civil.

D) Normas para el desarrollo de la guerra. Aunque es posible excluir el ataque directo a poblaciones civiles, resulta muy difícil garantizar la inmunidad de estas y la proporcionalidad de los "daños colaterales".

Por su parte, el Papa J uan Pablo II ha sido también rotundo en su "no a la guerra". Entre sus muchas declaraciones, suele citarse su discurso al cuerpo diplomático de 13 de enero de $2003^{32}$. Hablando de la guerra en general se afirma:

Ésta nunca es una simple fatalidad. Es siempre es una derrota de la humanidad. El derecho internacional, el diálogo leal, la solidaridad entre los Estados, el ejercicio tan noble de la diplomacia, son los medios dignos del hombre y las naciones para solucionar sus contiendas.

31 Nos referimos a las dos siguientes: una carta del Presidente de la Conferencia Episcopal Norteamericana al Presidente Bush (Letter to President Bush on Iraq. Bishop Wilton D. Gregory, September 13, 2002), publicada en Revista de Fomento Social 57 (2002), 535-539, y una declaración de la Conferencia Episcopal Norteamericana (Statement on Iraq. United States Conference of Catholic Bishops, Washington D. C., November 13, 2002). Este segundo documento colectivo hace suyos y desarrolla con más detalle los principales argumentos que había formulado en el primero a título personal el presidente de la Conferencia Episcopal.

32 Puede verse el texto en: Ecclesia (2003), n. 3.137 (25 enero), 18-20. 


\title{
Y pasando en seguida a la situación de Irak y a la amenaza de una intervención norteamericana, se dice:
}

\begin{abstract}
Sin repetir lo que os dije el año pasado en circunstancias parecidas, me conformaré con añadir hoy, ante el empeoramiento constante de la crisis medio-oriental, que su solución nunca podrá ser impuesta recurriendo al terrorismo o a los conflictosarmados, pensando que la solución consiste en victorias militares. Y, ¿qué decir de la amenaza de una guerra que podría recaer sobre las poblaciones de Irak, tierra de los profetas, poblaciones ya extenuadas por más de doce años de embargo? La guerra nunca es un medio como cualquier otro, al que se puede recurrir para solventar disputas entre naciones. Como recuerda la Carta de la Organización de las Naciones Unidas y el Derecho internacional, no puede adoptarse, aunque se trate de asegurar el bien común, si no es en casos extremos y bajo condiciones muy estrictas, sin descuidar las consecuencias para la población civil, durante y después de las operaciones.
\end{abstract}

El Papa está pensando también en la doctrina de la guerra justa, que ve de aplicación en el presente caso. Pero invoca además la necesidad de atenerse a las normas del Derecho internacional.

Otras muchas declaraciones de iglesias cristianas se podrían citar ${ }^{33}$. Como síntesis de todas ellas permítasenos citar unas palabras de Mr. Gregory, presidente de los obispos estadounidenses, cuando ya la ofensiva bélica sobre Irak era inminente ( 26 de febrero):

Nuestra conferencia de obispos continúa cuestionando la legitimidad moral de cualquier uso unilateral de la fuerza preventiva para derrocar el gobierno de Irak (...). Con los hechos que conocemos es difícil justificar el recurso a la guerra contra Irak, sin contar con la evidencia clara y adecuada sobre un ataque inminente de índole grave o de la participación de Irak en los ataques terroristas del 11 de septiembre ${ }^{34}$.

33 Algunas de especial interés, citadas por orden cronológico: "Irak: non à une guerreillégitime", Declaración de la Comisión Justicia y Paz de Francia (27 septiembre 2002); "La justicia, fundamento y condición de la paz", Declaración del Consejo Permanente de la Conferencia Episcopal Francesa (15 octubre 2002) [Ecclesia n. 3.141 (22 febrero 2003), 29]; "La guerra, camino sin retorno", Declaración de la Conferencia Episcopal de Inglaterra y País de Gales (15 noviembre 2002) [Ecclesia n. 3.141 (22 febrero 2003), 28]; “Ilegitimidad moral de una guerra preventiva", Declaración de la Conferencia Episcopal Alemana (20 enero 2003) [Ecclesia n. 3.144 (15 marzo 2003), 36]; "La paz, don de Dios e imperativo moral", Nota pastoral de la Comisión Permanente de la Conferencia Episcopal Española (19 febrero 2003) [Ecclesia n. 3.142 ( 1 marzo), 7]; "Dieser Krieg ist Ausdruck des Scheiterns der Politik", Declaración de los Presidentes de la Conferencia Episcopal y del Consejo de las Iglesias Cristianas de Alemania (20 marzo 2003).

34 Recogidas en Ecclesia (2003), n. 3.145 (22 marzo), 15. 


\section{Conclusión}

La doctrina moral sobre la guerra ha sufrido una importante evolución histórica, que ha tenido dos momentos significativos: la aparición de los Estados modernos y, ya en el siglo XX, las nuevas condiciones de la guerra moderna.

Si el primer momento llevó a una mayor precisión de la doctrina sobre la guerra justa hasta hacerla muy restrictiva, el siglo XX ha conducido a la convicción de que esa doctrina es prácticamente inaplicable, sobre todo si la decisión de desencadenar el conflicto se deja en manos de los Estados soberanos. Por eso, desde la creación de la Organización de las Naciones Unidas, esta responsabilidad se ha encomendado al Consejo de Seguridad de dicho organismo. Con esto no se renunciaba a la consideración moral de la guerra, reduciendo el tema a una pura cuestión de procedimiento legal, sino que se designaba a una instancia supraestatal como la única legitimada para aplicarla.

Sin embargo, las dificultades prácticas para que este órgano cumpliese en muchos casos su función ha reactualizado el concepto de intervención humanitaria y, en consecuencia, las motivaciones estrictamente éticas para justificar una acción armada en el territorio de otro Estado. En último término, es el derecho a la legítima defensa el que fuerza a no excluir de modo tajante el recurso a la violencia, por muchos problemas que plantee su aplicación.

En el caso de esta segunda intervención armada en Irak (marzo 2003), el gobierno de los Estados Unidos y los de sus aliados, ante la imposibilidad de encontrar el respaldo institucional del Consejo de Seguridad, han recurrido nuevamente a razones éticas: en primer lugar, al concepto de guerra preventiva y, de forma menos directa, a la intervención humanitaria.

Una consideración crítica de los argumentos aducidos difícilmente nos llevaría ajustificar la intervención. Lo que esta acción militar y las circunstancias que la han rodeado confirman es la necesidad de que sea un organismo supraestatal, y por tanto neutral, quien asuma la responsabilidad de la iniciativa bélica, aunque ello no exima del análisis ético de la cuestión. Ahora bien, esa necesidad se desdobla en otras dos, que nos son nuevas, pero hoy resultan más urgentes: la de una reforma en profundidad para la ONU, y la de un enfoque de estas grandes amenazas para la estabilidad mundial, no como problemas puntuales, sino en el marco de una propuesta global para construir la paz ${ }^{35}$.

35 En la línea de las excelentes sugerencias deV. FISAS, (2003), "Propuestas para construir la paz", El País (24 febrero), 7. 